\title{
Partitioning heritability analysis reveals a shared genetic basis of brain anatomy and schizophrenia
}

PH Lee, JT Baker, AJ Holmes, N Jahanshad, T Ge, J-Y Jung, Y Cruz, DS Manoach, DP Hibar, J Faskowitz, KL McMahon, Gl de Zubicaray, NH Martin, MJ Wright, D Öngür, R Buckner, J Roffman, PM Thompson and JW Smoller

Molecular Psychiatry (2017) 22, 1224; doi:10.1038/mp.2017.42; published online 21 February 2017

Correction to: Molecular Psychiatry advance online publication, 11 October 2016; doi:10.1038/mp.2016.164
The thirteenth author's name was presented incorrectly. It should have been listed as NG Martin.

\section{Elevated CYP2C19 expression is associated with depressive symptoms and hippocampal homeostasis impairment}

MM Jukić, N Opel, J Ström, T Carrillo-Roa, S Miksys, M Novalen, A Renblom, SC Sim, EM Peñas-Lledó, P Courtet, A Llerena, BT Baune, DJ de Quervain, A Papassotiropoulos, RF Tyndale, EB Binder, U Dannlowski and M Ingelman-Sundberg

Molecular Psychiatry (2017) 22, 1224; doi:10.1038/mp.2017.93; published online 18 April 2017

Correction to: Molecular Psychiatry (2017) 22, 1155-1163; doi: 10.1038/mp.2016.204; published online 29 November 2016

The ninth and eleventh authors' affiliations were presented incorrectly. They should have been linked to the following affiliations:
A Llerena - ${ }^{7} \mathrm{CICAB}$ Clinical Research Center, Extremadura University Hospital and Medical School, Badajoz, Spain. ${ }^{8} \mathrm{CIBER}-$ SAM, Madrid, Spain.

EM Peñas-Lledó - ${ }^{7} \mathrm{CICAB}$ Clinical Research Center, Extremadura University Hospital and Medical School, Badajoz, Spain. 